saisonalen Depression. Der Schlafforscher nannte Ergebnisse einer Studie mit 122 Patienten, die Fluoxetin, eine Lichttherapie, beides oder Placebo bekamen (Abb. 1) [Lam RW, et al. JAMA Psychiatry 2016; 73 (1): 56-63]. Die Lichttherapie wirkte dabei nicht schlechter als der SSRI, am stärksten gingen die Depressi- onen aber mit der Kombination von Antidepressivum und hellem Licht zurück.

$\mathrm{Zu}$ denken gibt nach Ansicht von Kunz auch eine Untersuchung zur ADHS-Prävalenz in den USA: Die ist ausgerechnet in den Bundesstaaten mit den meisten Sonnenstunden am geringsten. Das könne natürlich auch andere Ursachen ha- ben, es lohne sich aber, die Bedeutung der Lichtexposition nicht nur bei Depression, sondern auch bei anderen psychischen Störungen zu untersuchen.

Thomas Müller

Privatdozent Dr. Dieter Kunz: Schlafstörungen Psychiatrie Update, 11.-12.3.2016, Wiesbaden

\title{
Gesunder Mittagsschlaf? - Das hängt vom Alter und von der Dauer ab
}

\begin{abstract}
Bei Kindern scheint ein Nickerchen am Mittag gut fürs Lernvermögen zu sein. Bei älteren Menschen ist übermäßige Müdigkeit am Tage womöglich Zeichen einer beginnenden Demenz oder einer anderen schweren Erkrankung.
\end{abstract}

\begin{abstract}
E in Nickerchen am Nachmittag empE finden viele Menschen als erfrischend und erholsam. Tatsächlich haben Studien gezeigt, dass ein kurzer „PowerNap“ zu nachweisbaren Erholungseffekten führt und die Entscheidungsfähigkeit verbessert. Allerdings, so Privatdozent Dr. Dieter Kunz vom St.-HedwigKrankenhaus in Berlin, hat das Nickerchen wenig mit Schlaf im engeren Sinne zu tun. Häufig genügt ein wenige Minuten dauerndes Einnicken, um den Akku wieder aufzuladen. In dieser Zeit kommt es weder zu REM- noch zu Tiefschlaf, erläuterte der Somnologe. Von daher könnten die positiven Effekte auch nicht durch Mechanismen erklärt werden, wie sie beim Nachtschlaf auftreten.
\end{abstract}

\section{Tagesschlaf fürs Lernen wichtig}

Bei Kindern ist das offenkundig anders - sie schlafen mittags nicht nur tiefer und länger als Erwachsene, bei ihnen hat der Tagesschlaf teilweise ähnliche Funktionen wie der Nachtschlaf: Sie können das zuvor Gelernte viel besser im Gedächtnis behalten, auch fördert der Mittagsschlaf die emotionale Stabilität: Nach Studiendaten reagierten Kinder im Alter von zwei bis drei Jahren anschließend adäquater auf lösbare wie unlösbare Aufgaben, sagte Kunz. Er wies allerdings darauf hin, dass Kinder entspannt zu Bett gehen sollten. Zu viel Kortisol am Abend störe den Tiefschlaf. Der sei aber für die Gedächtniskonsolidierung gerade bei Kinder extrem wichtig: Nach in- tensivem Lernen werden ausgeprägte Tiefschlafphasen mit vermehrter DeltaAktivität beobachtet.

Bei Erwachsenen hängt der Nutzen des Mittagsschlafs offenbar mit der Dauer zusammen: Nach Daten einer Metaanalyse von elf Schlafstudien ist das Risiko für kardiovaskuläre Ereignisse bei einem regelmäßigen 30-Minuten-Nickerchen um rund $20 \%$ reduziert, danach steigt es jedoch linear an und ist bei einer Stunde pro Tag bereits um 20 \% erhöht [Yamada T et al. Sleep 2015; 38 (12): 1945 - 53]. Weitere Berechnungen deuten auf eine linear ansteigende Sterberate: Danach ist die Gefahr, innerhalb eines bestimmten Zeitraums zu sterben, bei einer Stunde Mittagsschlaf bereits um $20 \%$ erhöht. Hier muss jedoch klar zwischen Ursache und Wirkung unterschieden werden. Zwar, so Kunz, könne ein ausgeprägter Mittagsschlaf die zirkadiane Rhythmik stören und auf diese Weise nachts Ein- und Durchschlafstörungen begünstigen der Mittagsschlaf würde also nächtliche Schlafstörung aufrechterhalten, mit all den negativen Konsequenzen für die Gesundheit. Häufig sei der Mittagsschlaf bei älteren Menschen allerdings Ausdruck einer erhöhten Müdigkeit infolge bereits bestehender Erkrankungen. Der Somnologe verwies auf eine Studie mit 133 älteren Menschen mit erhöhtem Demenzrisiko [Cross N et al. J Sleep Res. 2015; 24: 494 - 502]. Von diesen gönnten sich $84 \%$ einen Mittagsschlaf. Solche
Personen waren überproportional häufig übergewichtig und von chronischen Erkrankungen betroffen, auch litten sie vermehrt an leichten kognitiven Störungen und Depressionen. Je häufiger und länger sie schliefen, umso schlechter stand es um ihre kognitiven Fähigkeiten. Ein neu auftretender Tagesschlaf könnte daher den Beginn einer chronischen oder altersbezogenen Erkrankung markieren.

\section{Erhöhtes Alzheimer-Risiko}

Vor allem der Zusammenhang mit einer Demenz ist interessant. Bei AlzheimerKranken ist der zirkadiane Rhythmus oft völlig gestört. Sie zeigen ein ähnliches Schlaf-Wach-Muster wie Nager, denen man den Nucleus suprachiasmaticus entfernt hat: einen fast stündlichen Wechsel von lebhafter Aktivität und Ruhe. Die damit verbundene nächtliche Unruhe zählt zu den stärksten Belastungsfaktoren für Angehörige und ist einer der Hauptgründe für die Einweisung in ein Pflegeheim. Eine Fragmentierung des Schlafs mit nächtlichen Schlafproblemen und zunehmenden Schlafphasen am Tag könnte daher auch Zeichen einer beginnenden Demenz sein. In einer Studie hatten kognitiv gesunde Menschen mit fragmentiertem Schlaf ein deutlich erhöhtes AlzheimerRisiko. Tierexperimente weisen auf einen Einfluss des orexinergen Transmittersystems. „Das ist von besonderer Bedeutung, da mehrere Orexinantagonisten in der Phase-III-Erprobung sind", sagte Kunz. Solche Substanzen könnten vielleicht auch den Nachtschlaf bei Demenzkranken verbessern und damit die nächtliche Unruhe mindern.

Thomas Müller

Privatdozent Dr. Dieter Kunz: Schlafstörungen. Psychiatrie Update, 11.-12.3.2016, Wiesbaden 